\title{
Geographische Tatsachen und Fiktionen in Platons Atlantisbericht
}

Rainer W. Kühne

Bürgerstr. 4, 38118 Braunschweig, Deutschland

kuehne70@gmx.de

Diese Abhandlung untersucht die geographische Beschreibung von Athen und Atlantis in Platons Dialogen Timaios und Kritias. Platon beschrieb sowohl Attika als auch die Athener Akropolis weitgehend korrekt. Ein Teil der Beschreibung ähnelt der Akropolis in mykenischer Zeit, anderes ist übertrieben, weiteres Platons Zeit entlehnt. Platons Beschreibung von Atlantis ist geologisch unmöglich. Griechische Elemente ab dem 7. Jh. v. Chr. flossen in die kulturelle Beschreibung von Atlantis ein. Die von Platon erwähnten Fluten und Kataklysmen beruhen weder auf griechischer noch ägyptischer Überlieferung, sondern auf seinen philosophischen Ideen.

Der sogenannte Atlantisbericht ist ein Bestandteil der Dialoge Timaios (20d-27a) und Kritias (106a-121c) des griechischen Philosophen Platon. Er beinhaltet eine Beschreibung nicht nur des Inselstaates Atlantis, sondern auch des urzeitlichen Athen.

Platon entstammte einer adligen Athener Familie. Als Sohn des Ariston und der Periktione wurde er 428/427 v. Chr. geboren. Bis zu dessen Tod im Jahre 399 v. Chr. war Platon acht Jahre lang ein Schüler und Anhänger des Sokrates. Er entwickelte die Idee eines Idealstaates, in dem Philosophenkönige regierten und der von Wächtern (Berufssoldaten) beschützt wurde. Diesen fiktiven Idealstaat beschrieb Platon in seinem Dialog Politeia. Im Jahre 388 v. Chr. reiste Platon nach Unteritalien und Sizilien und versuchte erfolglos, den Tyrannen Dionysios I von Syrakus zu einem Philosophenkönig zu erziehen. Platon besuchte Syrakus ein zweites Mal im Jahre 366 v. Chr. Wiederum scheiterte er dabei, Dionysios II zu einem Philosophenkönig zu erziehen. In den späteren Dialogen Timaios und Kritias versuchte Platon, den Idealstaat im Kriegsfall zu beschreiben (Tim. 19b-20c). Sprecher dieser Dialoge waren Sokrates, Timaios, Hermokrates und Kritias. Platon verstarb 348/347 v. Chr.

Der Athener Sokrates war der Begründer der attischen Philosophie. Als Sohn des Steinmetzen Sophroniskos und der Hebamme Phainarete wurde er um 470 v. Chr. geboren. In sämtlichen Dialogen Platons ist er einer der Redner, meist der Hauptredner. Sokrates wurde im März 399 v. Chr. wegen angeblicher Gotteslästerung durch Trinken aus dem Schierlingsbecher hingerichtet.

Der Timaios aus Platons Dialog ist historisch nicht bekannt und möglicherweise ein fiktiver Charakter. Platon schrieb, Timaios sei aus dem italischen Lokris gebürtig, stehe keinem seiner Landsleute an Vermögen und Herkunft nach, habe die höchsten Ämter und Ehrenstellen im Staate bekleidet, und in Allem, was philosophisches Streben heißt, das Höchste erreicht (Tim. 20a).

Demgegenüber stellte Platon die Sprecher Hermokrates und Kritias nur kurz vor. Sie seien philosophische und staatskluge Männer in Kampf und Schlachten (Tim. 19e).

Der historische Hermokrates war ein Staatsmann in Syrakus, der während des Peloponnesischen Krieges auf Seiten der Griechen Siziliens gegen Athen stand. Im Jahre 408/407 v. Chr. fiel er im Straßenkampf.

Der historische Kritias entstammte einer attischen Familie und war der Sohn des 
Kallaischros. Er war ein Vetter oder Bruder von Platons Mutter Periktione. Kritias war der Anführer der 30 Tyrannen von Athen. Im Jahre 404/403 v. Chr. fiel er im Kampf in Munichia.

Gemäß dem Dialog Timaios (20d-23d) und dem Dialog Kritias (113a) hat Kritias den Bericht über Atlantis und Athen durch Solon von einem ägyptischen Priester aus Sais erfahren.

Solon war ein athenischer Staatsmann und Dichter. Als Sohn des Exekestides wurde er um 640 v. Chr. geboren. Verstorben ist er 560/559 v. Chr.

Sais ist das heutige Sa el-Hagar, ein Ort im Nordwesten des Nildeltas. Psammetich gründete 664 v. Chr. die 26. (saitische) Dynastie, die bis zum Einbruch der Perser 525 v. Chr. regierte.

Soviel zu den Sprechern des Dialogs. Betrachten wir zunächst die Beschreibung Attikas und der Athener Akropolis.

Platon schrieb, die Grenzen Attikas haben sich damals bis an den Isthmos und gegen das übrige Festland bis zu den Höhen des Kithairon und des Parnes ausgedehnt, halten das Gebiet von Oropos zur Rechten und den Asopos zur Linken (Krit. 110d-e).

Mit dem Isthmos ist der Isthmos von Korinth gemeint. Es handelt sich um eine Landenge zwischen Mittelgriechenland und der Peloponnes. An seiner schmalsten Stelle ist er etwa sechs Kilometer breit. Bereits in mykenischer Zeit ist er von einer Mauer gesperrt worden.

Der Kithairon ist das bis zu 1408 Meter hohe westliche Grenzgebirge zwischen Attika und Boiotien. Noch heute ist er großenteils bewaldet.

Östlich schließt sich der noch heute gut bewaldete, bis zu 1413 Meter hohe Parnes an. Er ist ebenfalls ein Grenzgebirge zwischen Attika und Boiotien.

Oropos war eine Küstenstadt, deren Landschaft häufig zwischen Boiotien und Attika umstritten war und oft den Besitzer wechselte.

Der Asopos ist der Hauptfluss des südlichen Boiotien, entspringt im Gebiet des ehemaligen Ortes Leuktra und fließt in östlicher Richtung am Nordabhang des Kithairon, bis er bei Oropos ins Meer mündet.

Somit entspricht Platons Beschreibung der Grenzen des urzeitlichen Attika nahezu den Grenzen Attikas zu seiner Zeit. Befassen wir uns nun mit der Beschreibung der Athener Akropolis.

Gemäß Platon zog sich die Ausdehnung der Akropolis in früherer Zeit bis zum Eridanos und Ilisos hinab, fasste die Pnyx in sich und hatte der Pnyx gegenüber den Lykabettos zur Grenze (Krit. 111e-112a).

Der Eridanos war ein attischer Bach, der zu Platons Zeit das nördliche Athen durchfloss und in den Kephisos, den Hauptfluss Attikas, mündete.

Der llisos ist ein Fluss in Attika. Er entspringt am Nordwestabhang des Hymettos, floss am Südrand des antiken Athen vorbei, ist dort heute unterirdisch kanalisiert, mündet 
heutzutage in den Kephisos, in antiker Zeit aber möglicherweise in die Bucht von Phaleron.

Die Pnyx ist eine 109 Meter hohe, breite Felskuppe westlich der Akropolis. Sie wurde in antiker Zeit für die Volksversammlung umgebaut und lag am westlichen Rand der Stadt Athen.

Der Lykabettos ist ein 277 Meter hoher, steiler Bergkegel im heutigen Stadtgebiet von Athen, der aber östlich des antiken Athen stand.

Platon hat die Grenzen der urzeitlichen Akropolis so groß angegeben wie das historische Athen seiner Zeit. Bereits Thukydides (I 10) bemerkte aber, dass die Städte in mykenischer Zeit viel kleiner waren als zu seiner Zeit.

Platon beschrieb, wie die urzeitliche Athener Akropolis zerstört worden sein soll.

Eine besonders regnerische Nacht habe die Erde rings um die Akropolis aufgelockert und von ihr weggespült, indem zugleich Erdbeben und eine gewaltige Wasserflut, die dritte vor der Zerstörung zu Deukalions Zeit, entstanden waren (Krit. 112a).

Rhys Carpenter (1966) meinte hierin eine Erinnerung an Naturkatastrophen in mykenischer Zeit zu erkennen. Dagegen bezeichnete Hans Herter (1944) dies als legitime Hypothese Platons, der jedoch die Entwaldung durch Abholzung in mykenischer Zeit als Ursache verkannte.

Ferner schrieb Platon, es habe damals nur eine einzige Wasserquelle gegeben, wo jetzt die Akropolis steht. Nach deren Versiegen in Folge von Erdbeben seien nur noch die kleinen Wässerchen von ihr übrig geblieben, welche sich rings um die Akropolis herumziehen (Krit. 112c-d).

Tatsächlich hat Oskar Broneer (1939) einen Brunnen aus mykenischer Zeit nachgewiesen, der etwa 25 Jahre in Gebrauch blieb und an der Grenze späthelladisch III B/C etwa 1200 v. Chr. durch ein Erdbeben verschüttet wurde.

Ferner schrieb Platon, die Höhe selbst war um das Heiligtum der Athene und des Hephaistos herum von den Kriegern für sich in Besitz genommen worden, indem sie dasselbe mit einer einzigen Mauer umgeben hatten, so bewohnten sie den nördlichen Teil der Akropolis (Krit. 112b).

Tatsächlich hat Oskar Broneer (1948) nachgewiesen, dass in mykenischer Zeit sich die Behausungen der Krieger im nördlichen Teil der Akropolis befanden.

Ferner schrieb Platon, die Athener seien hinsichtlich der Schrift immer eben erst eingerichtet, wenn die Regenflut des Himmels über sie hereinbricht und nur die der Schrift Unkundigen und Ungebildeten übrig lässt (Tim. 23a-b).

Rhys Carpenter (1966) verwies darauf, dass die mykenischen Griechen schriftkundig waren. Ihre Schriftkundigkeit wurde von Michael Ventris und John Chadwick (1953) bewiesen, indem sie die Sprache des Linear B als frühgriechischen Dialekt identifizierten.

Tatsächlich könnte diese Beschreibung der Akropolis, der Zerstörungen und des Verlustes der Schriftkenntnis eine Beschreibung attischer Zustände am Ende der mykenischen Zeit 
nahelegen. Zu beachten ist jedoch, dass Platon behauptete, ein urzeitliches Athen vor 9000 Jahren zu beschreiben (Krit. 111a).

Kommen wir jetzt zu Platons Beschreibung der Geographie von Atlantis.

Damals war das atlantische Meer befahrbar, denn vor der Mündung, welche Säulen des Herakles heißt, hatte es eine Insel, welche größer war als Asien und Libyen zusammen, und von ihr konnte man damals auf das gegenüberliegende Festland, welches jenes recht eigentlich so zu nennende Meer umschließt, hinübersetzen. Das, was sich innerhalb der eben genannten Mündung befindet, erscheint wie eine Bucht mit einem engen Eingang, jenes Meer aber kann in Wahrheit also und das es umgebende Land mit vollem Fug und Recht Festland heißen (Tim. 24e-25a).

Die Insel Atlantis lag jenseits der Säulen des Herakles, war größer als Libyen und Asien zusammen, ist aber durch Erdbeben untergegangen (Krit. 108e).

Gadeiros erhielt den äußersten Teil der Insel, gegenüber den Säulen des Herakles bis zum gadeirischen Land, nach ihm ist auch dieses Land benannt (Krit. 114b).

Eindeutig handelt es sich bei den Säulen des Herakles um die Straße von Gibraltar (Gisinger 1933). Das gadeirische Land ist die Gegend um Cadiz, wobei Gadir ein phönizisches Wort ist und Umzäunung bedeutet (Plinius IV 120). Das atlantische Meer meint nicht nur den Atlantischen Ozean, sondern den Okeanos, der Europa, Asien und Libyen umfließt. Das inn umgebende Festland ist somit nicht Amerika, sondern der Erdrand der Erdscheibe.

Aufgrund der Angaben Platons vermuteten S. Mitchell $(1877)$, Pierre Termier $(1913,1915)$ und William Babcock (1917), die Insel Atlantis sei eine kontinentgroße Insel im Nordatlantik gewesen. Diese Ansicht wurde bereits von Alfred Wegener (1912) und Charles Schuchert (1917a) widerlegt, die erkannten, dass Meeresboden und Kontinentalboden geologisch verschieden sind, so dass niemals später versunkene Landbrücken oder Inselkontinente im Atlantik existiert haben können. (Auch Pierre Termiers $(1913,1915)$ Argument, das in der Tiefsee bei den Azoren geborgene Tachylith könne nur bei Atmosphärendruck in der Luft entstanden sein, konnte widerlegt werden. Charles Schuchert (1917b) und Rudolph Schuller (1917) wiesen nach, dass Tachylith auch in Meerwasser entstehen kann.)

Vielmehr entsprechen diese Größenangaben der Philosophie Platons. Im Phaidon (109ab) schrieb er, das Mittelmeer sei wie ein Teich, um den die griechischen Städte wie Frösche säßen, wohingegen die wahre Erde viel größer sei als alle bekannten Länder.

Auch die Beschreibung der kulturellen Höhe von Atlantis ist keinesfalls urzeitlich, auch nicht bronzezeitlich, sondern entspricht Platons Zeit, frühestens aber seit dem 7. Jh. v. Chr.

So schrieb Platon, die Atlanter hätten Trireme besessen (Krit. 117d).

Gemäß Thukydides (I 13) haben die Korinther als erste die hellenischen Trireme gebaut. Der Korinther Ameinokles baute vier Trireme für die Samier. Er ging etwa 300 Jahre vor dem Ende des Peloponnesischen Krieges (Kapitulation Athens 404 v. Chr.) nach Samos. Der erste Kriegseinsatz der Trireme war der zwischen Korinth und Korkyra etwa 40 Jahre später. 
Ferner schrieb Platon, die Atlanter hätten Hoplitai besessen (Krit. 119b).

Die Hoplitai waren das schwer bewaffnete Fußvolk der griechischen Phalanx. Die Bewaffnung bestand aus Lanze, Schwert, Schild, Helm, Panzer und Beinschienen. Die Bezeichnung Phalanx in der Bedeutung einer mehr breiten als tiefen militärischen Aufstellung verwendete zuerst Xenophon. Ihre Anfänge reichen in das 7. Jh. v. Chr. zurück.

Gemäß Platon bildeten die Atlanter den Gott Poseidon ab, wie er auf seinem Wagen stehend, sechs geflügelte Rosse lenkt, und so groß dargestellt war, dass das Haupt seines Bildnisses die Decke des Tempels berührte (Krit. 116d-e). Ferner gab es Stiere im Heiligtum des Poseidon, die ihm geopfert wurden (Krit. 119d-e).

Der einzige Meeresgott, der zu Pferden und Stieren eine enge Beziehung hat, ist der griechische Poseidon. Er zeugte den Areion und den Pegasos, mit inm ist das trojanische Pferd verbunden, inm wurden Pferde und Stiere geopfert.

Außerdem erinnert das obengenannte Bildnis an den olympischen Zeus. Pheidias war als Bildhauer von etwa 460 bis etwa 430 v. Chr. tätig. Den Alten schien sein 12 Meter hohes Bildnis des sitzenden Zeus in Olympia, als erhöbe es sich, um die Decke zu sprengen.

Zuletzt sei auf zwei Passagen verwiesen, die auf Platons Philosophie der urzeitlichen Kataklysmen und seiner versuchsweisen Erklärung einiger Mythen als Naturereignisse verweisen.

So schrieb Platon, der Phaethon-Mythos habe eine astronomische Erklärung (Tim. 22c-d). Ähnlich äußerte er auch im Politikos (270c-d), einige Mythen beruhten auf astronomischen Vorgängen.

Ferner schrieb Platon, mehrere Fluten (Tim. 23b) und Naturkatastrophen (Tim. 22c, 23a-b) hätten während der Menschheitsgeschichte stattgefunden. Auch im Politikos (268e-274d) und in Nomoi (III 679d) erwähnte er mehrere angebliche Kataklysmen während der Menschheitsgeschichte.

Zusammenfassend erinnert Platons Beschreibung des urzeitlichen Athen zwar an das historische Athen der mykenischen Zeit, dürfte aber eher seiner Beobachtungsgabe und Vorstellungskraft entspringen. Seine Beschreibung von Atlantis ist geologisch unmöglich, die angebliche Höhe der Kultur von Atlantis ist keineswegs urzeitlich, sondern vergleichbar mit der Griechenlands zur Zeit Platons. Einige Elemente der Atlantis-Geschichte entstammen Platons Philosophie.

Babcock, William H.: "Atlantis and Antilla", The Geographical Review 3 (1917) 392-395.

Broneer, Oskar: "A Mycenaean Fountain on the Athenian Acropolis", Hesperia 8 (1939) 317-429.

Broneer, Oskar: "What Happened at Athens?", American Journal of Archaeology 52 (1948) 111-124.

Carpenter, Rhys: Discontinuity in Greek Civilization. Cambridge University Press (1966). 
Gisinger, F.: "Zur geographischen Grundlage von Platons Atlantis", Klio 26 (1933) 32-38.

Herter, Hans: "Altes und Neues zu Platons Kritias", Rheinisches Museum für Philologie 92 (1944) 236-265.

Mitchell, S.: "The Lost Atlantis and the Challenger Soundings", Nature 15 (1877) 553-556.

Schuchert, Charles: "Atlantis and the Permanency of the North Atlantic Ocean Bottom", Proceedings of the National Academy of Sciences 3 (1917a) 65-72.

Schuchert, Charles: "Atlantis, the Lost Continent - A Review of Termier's Evidence", The Geographical Review 3 (1917b) 64-66.

Schuller, Rudolph: "Atlantis, the Lost Continent - A Review of Termier's Evidence", The Geographical Review 3 (1917) 61-64.

Termier, Pierre: "L'Atlantide", Bulletin de l'Institute Océanographique de Monaco 256 (1913) 11-146.

Termier, Pierre: "Atlantis", Annual Report of the Smithsonian Institution (1915) 219-234.

Ventris, Michael und Chadwick, John: "Evidence for Greek Dialect in the Mycenaean Archives", Journal of Hellenic Studies 73 (1953) 86-103.

Wegener, Alfred: "Die Entstehung der Kontinente", Geologische Rundschau 3 (1912) 276292. 